\title{
Study of Trichomonas vaginalis Infection in Women Visiting Health Centers in Bahar City and Determination of Metronidazole Susceptibility of the Isolated Parasites
}

\author{
Leila Morshedloo', Mohammad Fallah², Amir Hossein Maghsood ${ }^{3}$, Mohammad Matini4,"* \\ ${ }^{1}$ MSc in Parasitology, Hamadan University of Medical Sciences, Hamadan, Iran \\ ${ }^{2}$ Professor, Department of Medical Parasitology and Mycology, School of Medicine, Hamadan University of Medical \\ Sciences, Hamadan, Iran \\ ${ }^{3}$ Associate Professor, Department of Medical Parasitology and Mycology, School of Medicine, Hamadan University of \\ Medical Sciences, Hamadan, Iran \\ ${ }^{4}$ Assistant Professor, Department of Medical Parasitology and Mycology, School of Medicine, Hamadan University of Medical \\ Sciences, Hamadan, Iran
}

* Corresponding Author: Mohammad Matini, Department of Medical Parasitology and Mycology, School of Medicine, Hamadan University of Medical Sciences, Hamadan, Iran.Email: matini@umsha.ac.ir

\begin{tabular}{|c|c|}
\hline \multirow[b]{2}{*}{$\begin{array}{l}\text { Received: } 19.09 .2017 \\
\text { Accepted: } 15.01 .2018\end{array}$} & Abstract \\
\hline & \multirow{6}{*}{$\begin{array}{l}\text { Background and Objective: Trichomoniasis is one of the most common } \\
\text { non-viral sexually transmitted infections worldwide. Thus, we aimed to } \\
\text { determine the prevalence of this infection among women in Bahar city, Iran, } \\
\text { and evaluate drug susceptibility of the isolated parasites. } \\
\text { Materials and Methods: This cross-sectional study was conducted on } 850 \\
\text { females visiting the health centers of Bahar city, during } 2016 \text {. The diagnosis } \\
\text { of Trichomonas vaginalis infection was carried out by using wet mount and } \\
\text { Dorset culture medium. In the next step, the obtained parasites were } \\
\text { transferred to Diamond Medium and after the preparation of their pure } \\
\text { cultures, they were placed under laboratory and aerobic conditions to test } \\
\text { their drug sensitivity. } \\
\text { Results: Four ( } 0.5 \%) \text { isolates of } T \text {. vaginalis were obtained from the } \\
\text { participants. Drug sensitivity of the isolates after } 48 \mathrm{~h} \text { ranged between } 1.6 \\
\text { and } 25 \mu \mathrm{g} / \mathrm{ml} \text {, with the mean and standard deviation of } 12.6 \pm 8.26 \mu \mathrm{g} / \mathrm{ml} \text {. } \\
\text { Conclusion: We found that the prevalence of trichomoniasis in the studied } \\
\text { women in this region is lower than most parts of Iran. Due to the importance } \\
\text { of this infection, this prevalence rate is significant and needs proper } \\
\text { diagnosis and treament. }\end{array}$} \\
\hline \multirow{6}{*}{$\begin{array}{l}\text { How to Cite this Article: } \\
\text { Morshedloo L, Fallah M, } \\
\text { Maghsood AH, Matini M. Study } \\
\text { of Trichomonas vaginalis Infe- } \\
\text { ction in Women Visiting Health } \\
\text { Centers in Bahar City and } \\
\text { Determination of Metronidazole } \\
\text { Susceptibility of the Isolated } \\
\text { Parasites. Avicenna J Clin Med. } \\
2018 ; 24(4): 315-321 \text {. DOI: } \\
10.21859 \text { ajcm.24.4.315. }\end{array}$} & \\
\hline & \\
\hline & \\
\hline & \\
\hline & \\
\hline & $\begin{array}{l}\text { Keywords: Epidemiology, Metronidazole, Parasitic Sensitivity Test, } \\
\text { Trichomonas vaginalis }\end{array}$ \\
\hline
\end{tabular}




\title{
بررسى فراوانى آلودكى به تريكوموناس وازيناليس در زنان مراجعه كننده به مراكز بهداشتى - درمانى شهر بهار و تعيين ميزان حساسيت به مترونيدازول در انكلهاى جداشده
}

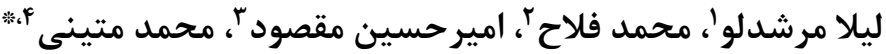

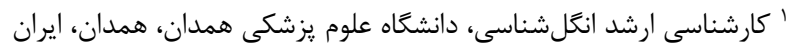

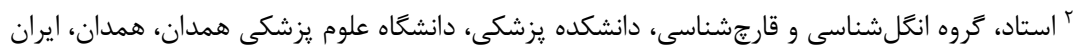

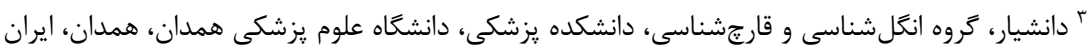

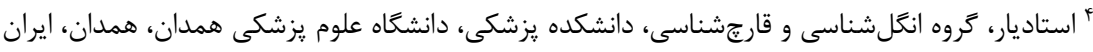

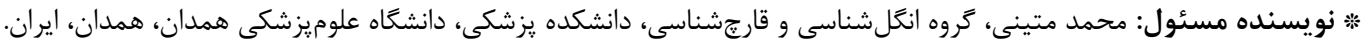
ايميل: matini@umsha.ac.ir

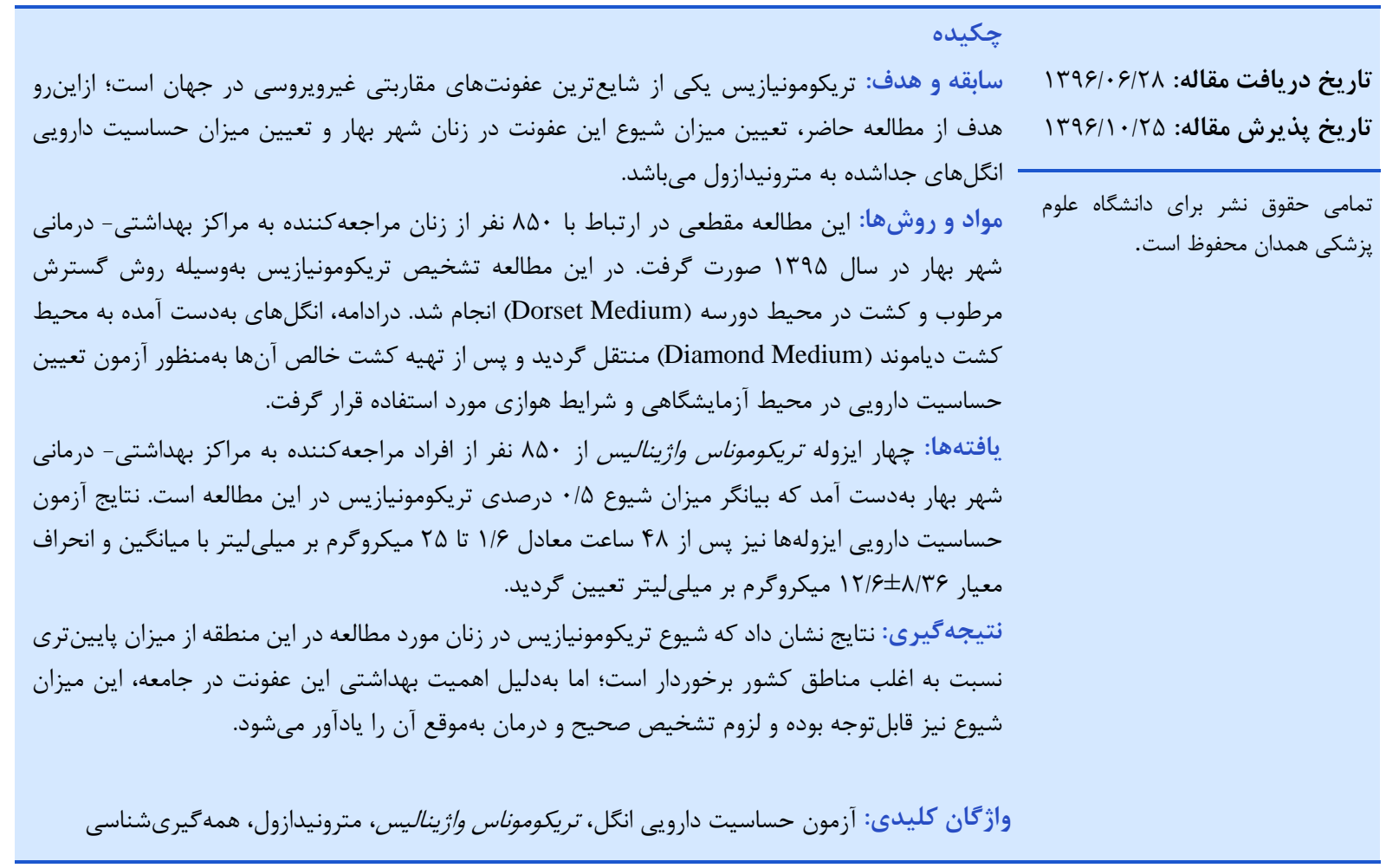

عفونت ناشى از اين انكل در زنان باردار مىتواند عوارضى از قبيل

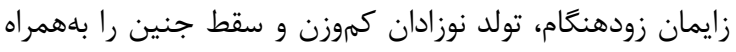

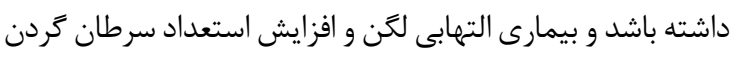

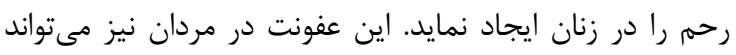

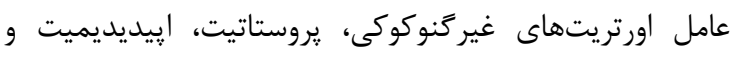

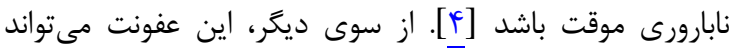

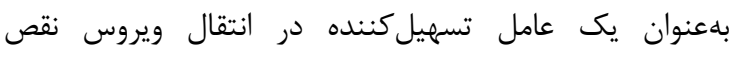
ايمنى انسان (HIV: Human Immunodeficiency Virus)
تريكوموناس وازيناليس تكياختهى ساكن مجارى ادرارى-

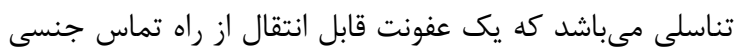

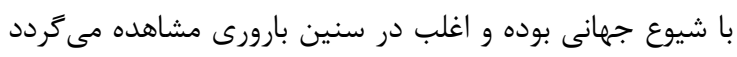

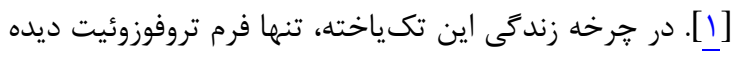

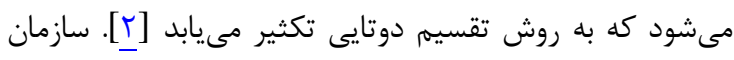
جهانى بهداشت (WHO: World Health Organization)

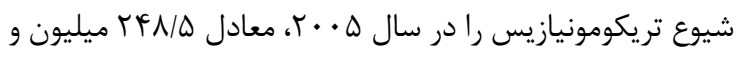

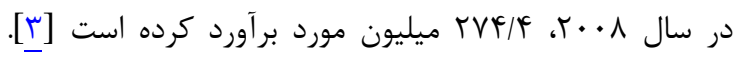


كسترش مرطوب تهيه شد و بdمنظور تشخيص تريكوموناس

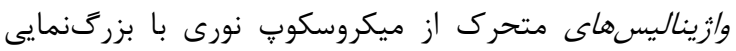

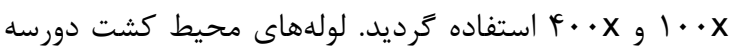

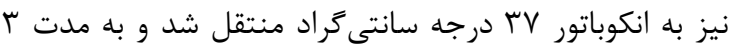

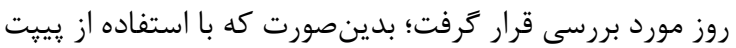

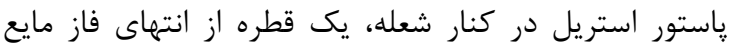

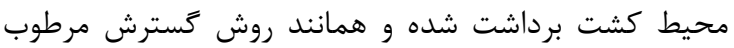
آزمايش شد [q].

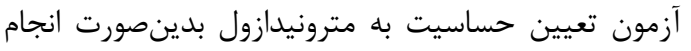

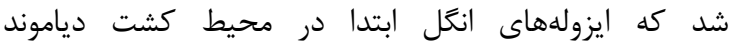
بdصورت خالص كشت داده شدند [ [1]

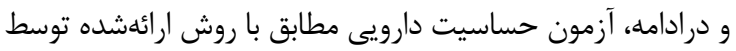
Meingassner (CDC: Centers for Disease Control and Prevention)

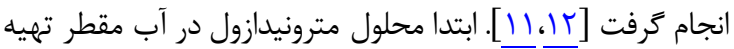

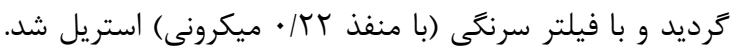
سيس، غلظتهايى از دارو در محيط كشت دياموند با روش

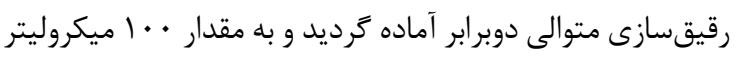

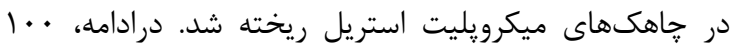

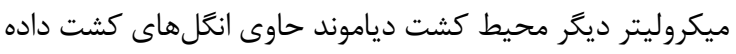

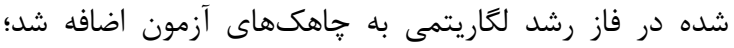

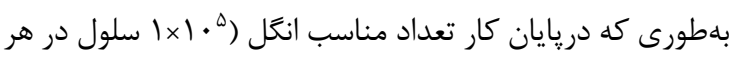

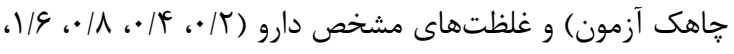
lr/ ميلى ليتر ) حاصل شد. درانتها، ميكرويليتهاى آزمون در انكوباتور

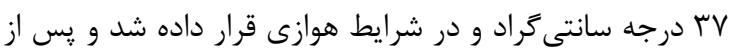

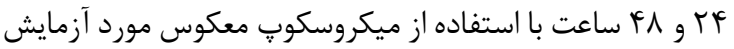

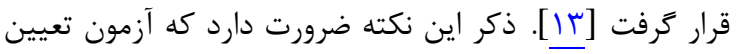

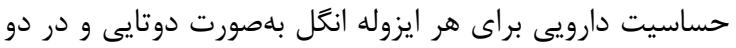
زمان مختلف تكرار زرديد.

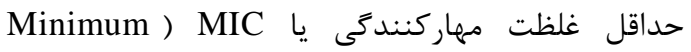
(Inhibitory Concentration از دارو كه يس از ^^ץ ساعت انكوباسيون سبب از حركت افتادن

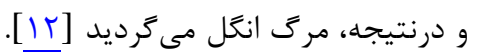
تجزيه و تحليل آمارى دادهها نيز با استفاده از نرمافزار SPSS 16

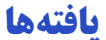

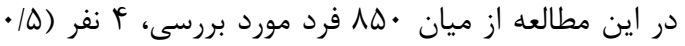

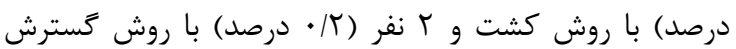

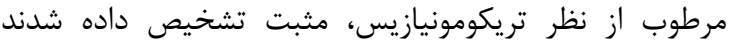

برمبناى نتايج، ميزان حساسيت روش گسترش مرطوب در

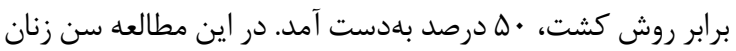

و ساير عفونتهاى قابل انتقال از راه جنسى عمل نمايد. شايان

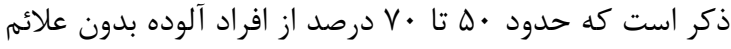

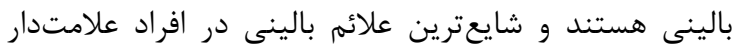
عبارت است از: ترشح، خارش و سوزش وازٔن كه اين علائم در

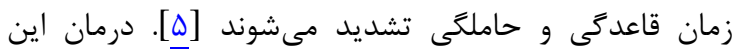

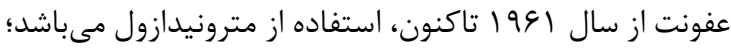

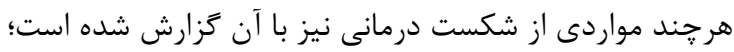
بلهطورى كه مركز كنترل و ييشكيرى از بيمارىها در آر آمريكا،

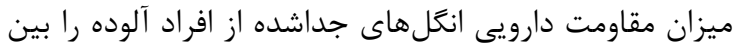

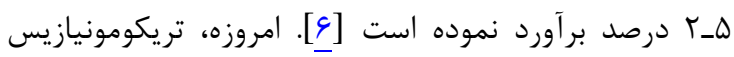
بيش از كذشته مورد توجه قرار گرفته است. مطالعات نشان

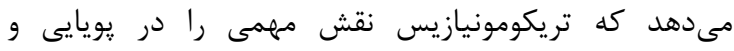

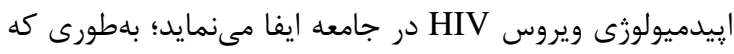
اين عفونت مى تواند باعث افزايش ابتلا و انتقال ويروس

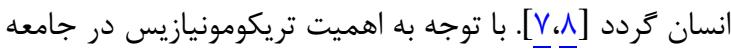

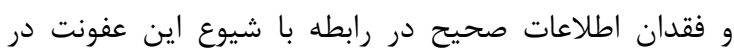

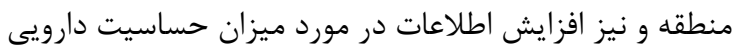

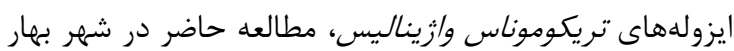

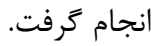

\section{مواد و روشها}

در اين مطالعه توصيفى- مقطعى، • • م نفر از زنان

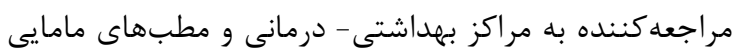

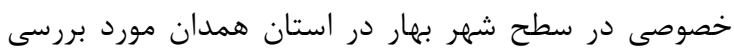

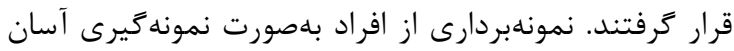

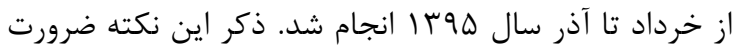

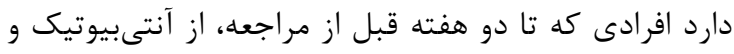

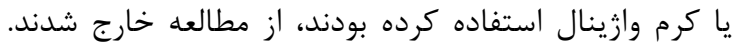

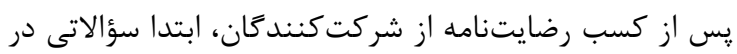

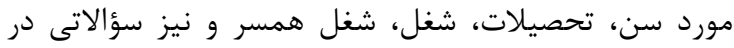

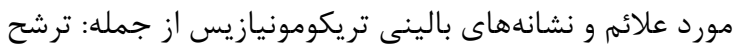

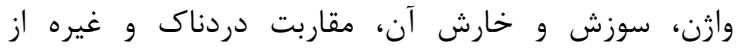

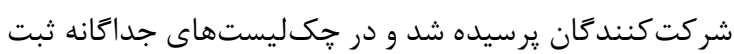
كرديد. نمونه كيرى بدينصورت انجام شد كه ابتدا توسط ماما يا

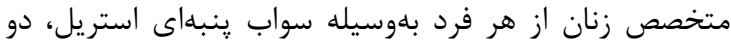

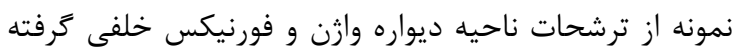

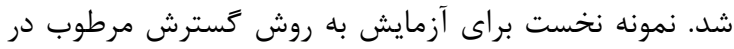

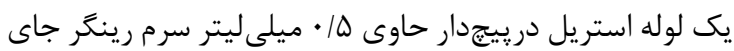

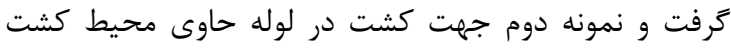

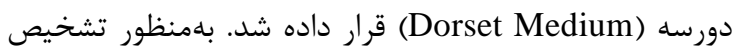
انغل و جلوگيرى از بى حركتشدن آن، نمونه آناى گرفتهشده

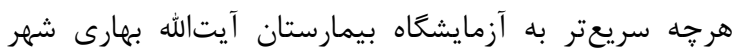

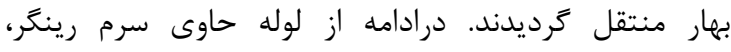




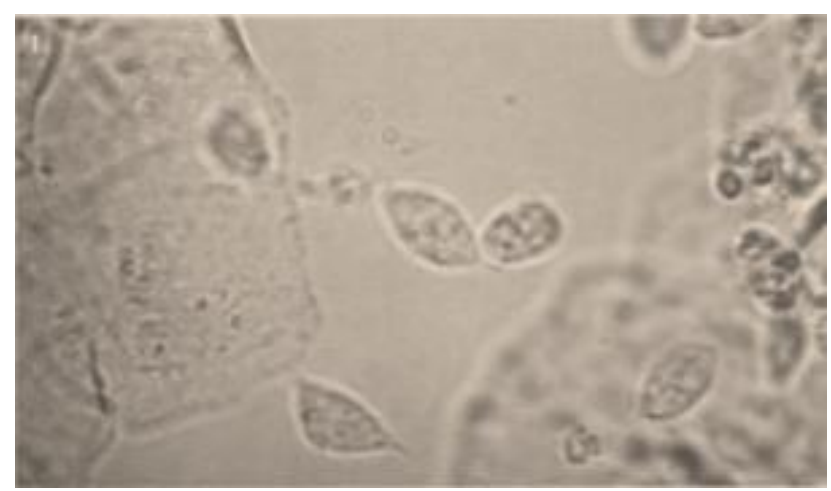

شكل ا: انتل تريكوموناس واريناليس در كّترش مرطوب تهيهشده از نمونه واثن

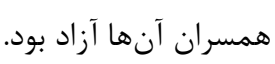

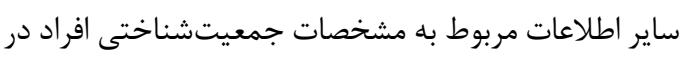

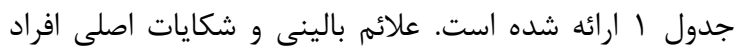

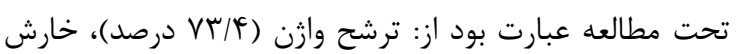

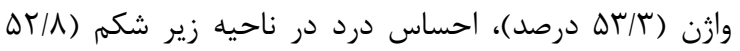

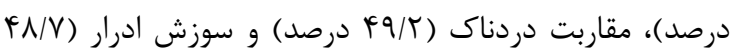

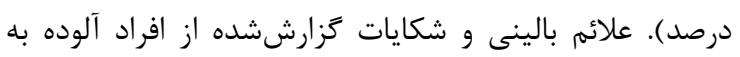

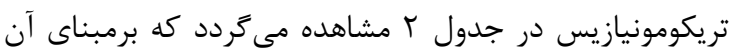

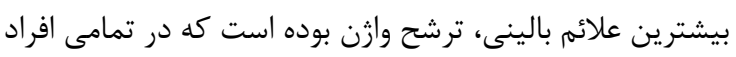
آلوده مشاهده كرديده است.

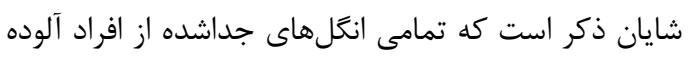

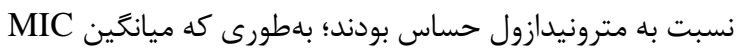

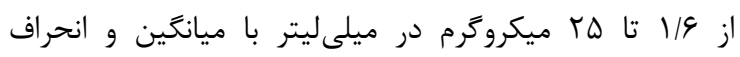

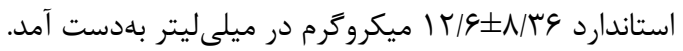

شركت كننده در محدوده ها تا DF سال قرار داشت و اغلب

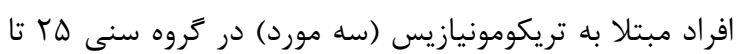

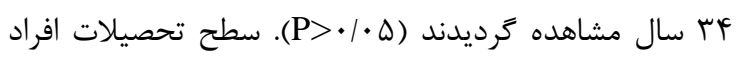

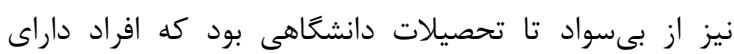

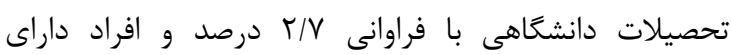

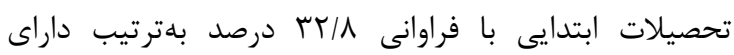

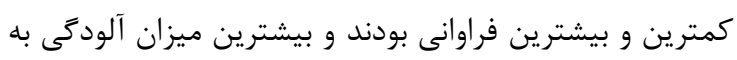

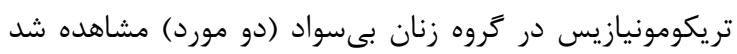

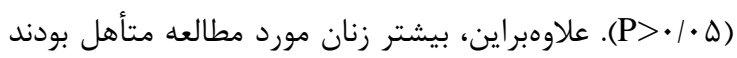
( ش

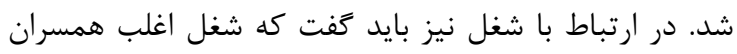

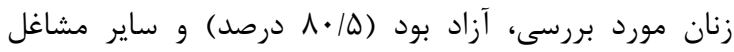

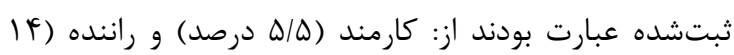

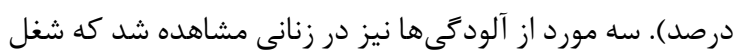

جدول ا: توزيع فراوانى افراد شركت كننده در مطالعه بر حسب سن، تحصيلات، شغل و محل سكونت

\begin{tabular}{|c|c|c|c|c|}
\hline \multirow{2}{*}{ 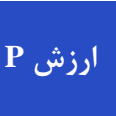 } & \multicolumn{4}{|c|}{ آلودَّى به تريكوموناس وازيناليس } \\
\hline & جمع، تعداد (درصد) & موارد منفى، تعداد (درصد) & موارد مثبت، تعداد (درصد) & \\
\hline \multirow{5}{*}{$>\cdot 1 \cdot \Delta$} & & & & سن، سال \\
\hline & $(1 \cdots) 99$ & $(1 \cdots) 99$ & $(\cdot) \cdot$ & DQ_KF \\
\hline & (1..) TEG & $(१ ৭ / r) r \Delta q$ & $(\cdot \mid \Lambda) r$ & rQ_rF \\
\hline & $(1 \cdots)$ YFE & $(१ ৭ / 9) T F \Delta$ & $(\cdot / 4) 1$ & $r \Delta_{-} F F$ \\
\hline & r (l. & (l. (1) & $(\cdot) \cdot$ & $r \Delta \Delta_{-} \Delta f$ \\
\hline \multirow{6}{*}{$>\cdot 1 \cdot \Delta$} & & & & تحصيلات \\
\hline & $(1 \cdots) \wedge r$ & $(9 \vee / 9) \wedge$. & $(r / Y) T$ & بىسواد \\
\hline & $(1 \cdots)$ rVq & $(99 / 9)$ TVA & $(\cdot \mid f) 1$ & ابتدايى \\
\hline & $(1 \cdot) 190$ & $(99 / 0) 194$ & $(\cdot \mid \Delta) 1$ & راهنمايى \\
\hline & $(1 \cdots)$ rVI & $(1 \cdots)$ TVI & $(\cdot) \cdot$ & دبيرستان \\
\hline & rr ( ( ) & rr ( ( ) & $(\cdot) \cdot$ & دانشخاهى \\
\hline \multirow{3}{*}{$>\cdot 1 \cdot \Delta$} & & & & شغل \\
\hline & $(1 \cdots) \wedge 10$ & $(99 / \Delta) \wedge 11$ & $(\cdot \mid Q)^{f}$ & خانهدار \\
\hline & $(1 \cdots) r \Delta$ & $(1 \cdot \cdots) r \Delta$ & $(\cdot) \cdot$ & كارمند \\
\hline \multirow{3}{*}{$>\cdot 1 \cdot \Delta$} & & & & محل سكونت \\
\hline & $(1 \cdot \cdots)$ VTG & $(99 / D) V T r$ & $(\cdot \mid \omega)^{f}$ & شهر \\
\hline & $(1 \cdots) \mid r F$ & $(1 \cdots) \mid r F$ & $(\cdot) \cdot$ & 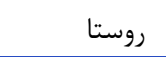 \\
\hline
\end{tabular}


جدول ب: علائم بالينى گَزارش شده در افراد مبتلا به تريكومونيازيس

\begin{tabular}{|c|c|c|c|}
\hline جمع، تعداد (درصد) & فاقد به تريكومنم، تعداد (درصد وازيناليس & داراى علائم، تعداد (درصد) & علائم بالينى \\
\hline$(1 \cdots) f$ & $(\cdot) \cdot$ & $(1 \cdots)^{f}$ & ترشح واثن \\
\hline$(1 \cdots)^{r}$ & $(r \Delta) 1$ & $(V \Delta) r$ & قوام غيرطبيعى ترشح \\
\hline$(1 \cdot \cdot)^{k}$ & $(r \Delta) 1$ & $(V \Delta) r$ & مقاربت دردناى \\
\hline$(1 \cdots)^{f}$ & $(r \Delta) 1$ & $(V \Delta) r$ & درد در ناحيه تحتانى شكم \\
\hline$(1 \cdots)^{r}$ & $(\Delta \cdot) T$ & $(\Delta \cdot) T$ & خارش در ناحيه تناسلى \\
\hline$(1 \cdots)^{r}$ & $(\Delta \cdot) r$ & $(\omega \cdot) r$ & سوزش در ناحيه تناسلى \\
\hline$(1 \cdots)^{f}$ & $(V \Delta) r$ & $(r Q) 1$ & سوزش ادرار \\
\hline$(1 \cdot \cdot)^{f}$ & $(V \Delta) r$ & $(r \Delta) 1$ & تكرر ادرار \\
\hline$(1 \cdots) f$ & $(V \Delta) r$ & $(r \Delta) 1$ & ترشح زرد مايل به سبز \\
\hline
\end{tabular}

پايين عفونت در ميان زنان در اين منطقه از كشور مىباشد.

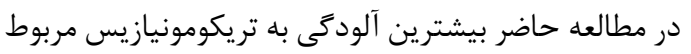

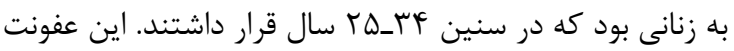

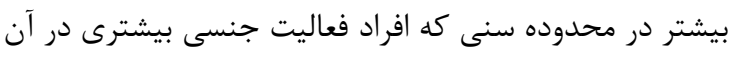

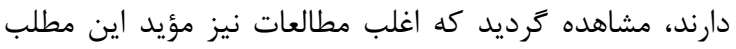

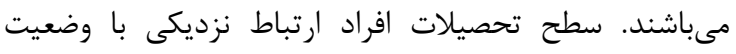

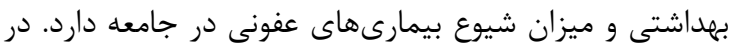

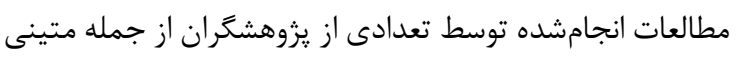

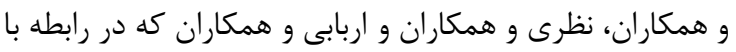

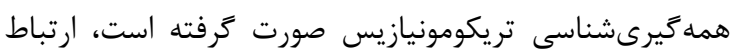

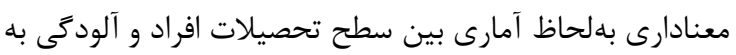

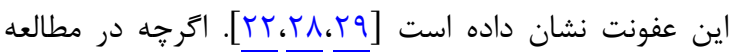

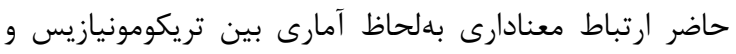

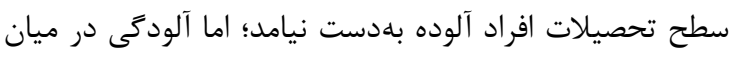

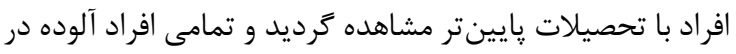

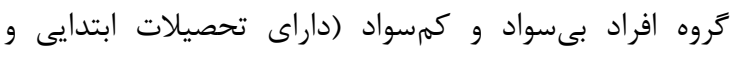

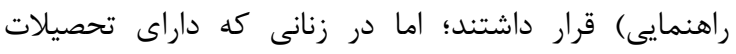

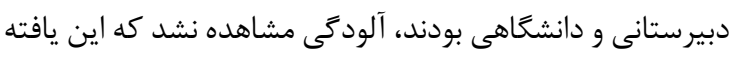

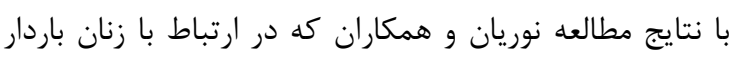

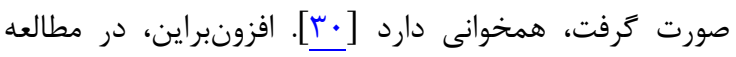

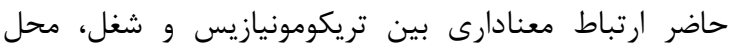
سكونت و شغل همسر مشاهده نشد كه اين نتايج با يافتههاى مطالعه متينى و همكاران كه در شهر قروه انجام گرفت، همخوان مىباشد [YT].

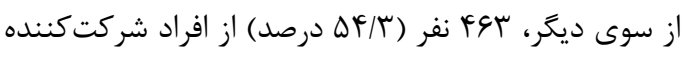

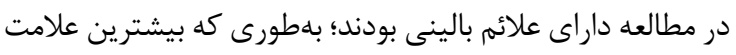

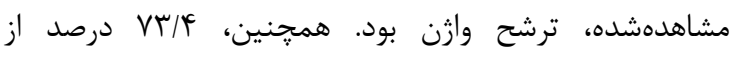
شركت كنند

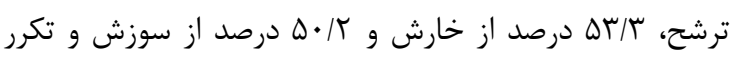

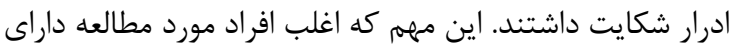

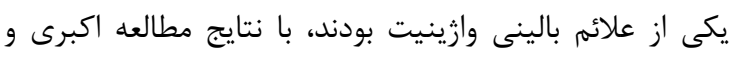

عوامل فرهنگیى، اجتماعى و بهداشتى در جوامع مختلف بر

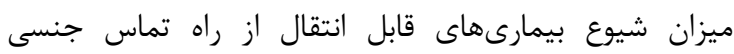

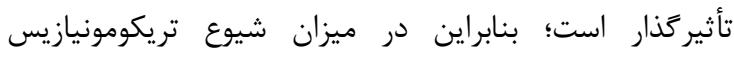

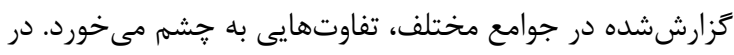

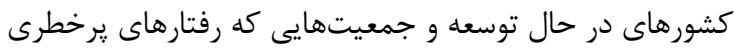

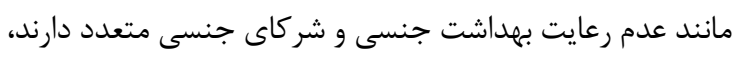

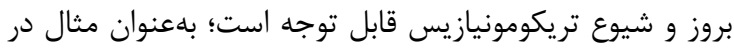

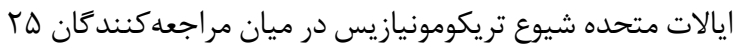

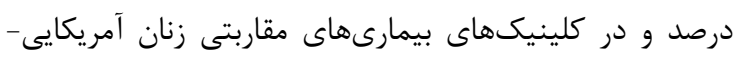

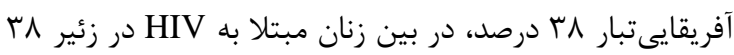

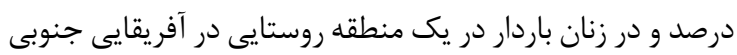

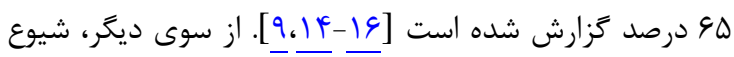

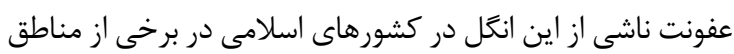

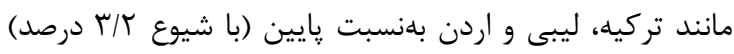

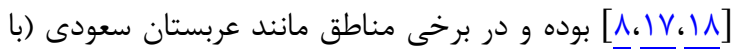

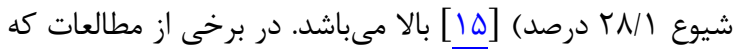
از روش PCR جهت تشخيص عوامل وازينيت استفاده شده است،

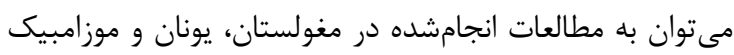

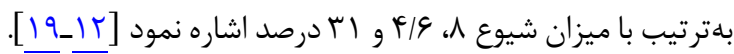

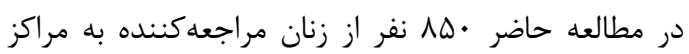

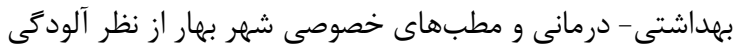

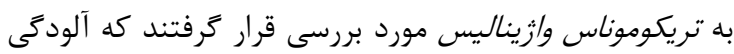

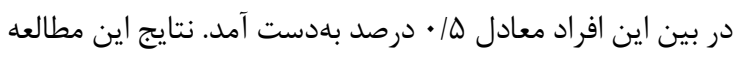

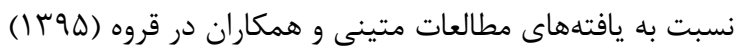

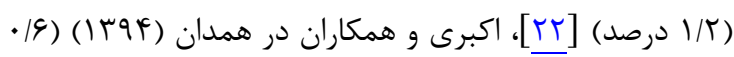

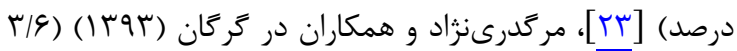

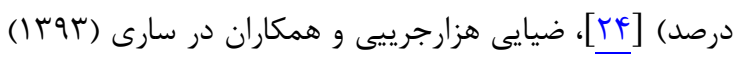

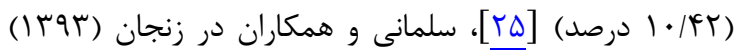
(

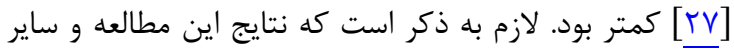

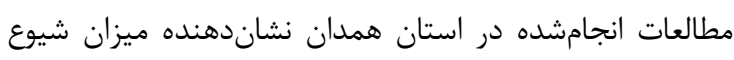


بلهور كلى، با درنظركرفتن نتايج بررسى ها در شهرهاى ديكر،

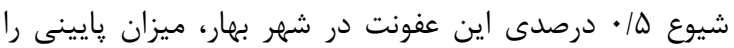

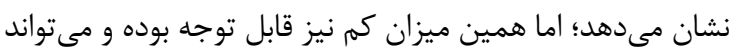

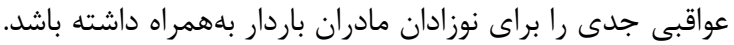

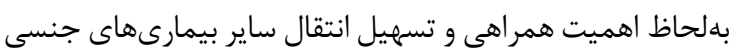

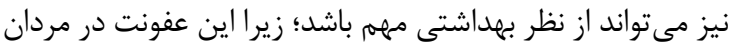

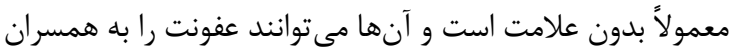

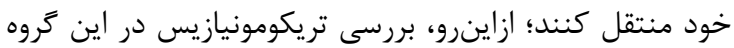
از افراد نيز حائز اهميت است.

\section{تشكر و قلر داذى}

اين مقاله بركرفته از يايانامه مقطع كارشناسى ارشد رشته

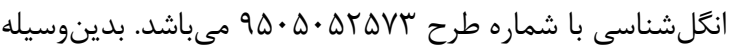

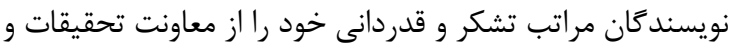

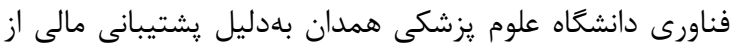

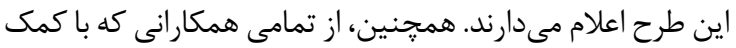

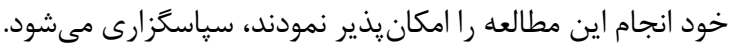
ذكر اين نكته ضرورت دارد كه هيج حاضر زَارش نتخرديد.

\section{REFERENCES}

1. French L, Horton J, Matousek M. Abnormal vaginal discharge: using office diagnostic testing more effectively. $J$ Fam Pract. 2004;53(10):805-14. PMID: 15469777

2. Warren KS, Mahmoud AA. Tropical and geographical medicine. New York, NY: McGraw-Hill; 1990.

3. World Health Organization. Global incidence and prevalence of selected curable sexually transmitted infections-2008. Geneva: Reproductive Health Matters; 2012.

4. Cudmore SL, Delgaty KL, Hayward-McClelland SF, Petrin DP, Garber GE. Treatment of infections caused by metronidazole-resistant Trichomonas vaginalis. Clin Microbiol Rev. 2004;17(4):783-93. PMID: 15489348 DOI: 10.1128/CMR.17.4.783-793.2004

5. Habibpour R, Amirkhani A. Matinnia N. Contamination rate of Trichomonas vaginalis in females referring to Taamin Ejtemayi hospitals in Hamedan in 2005. Zahedan J Res Med Sci. 2007;8(4):245-51.

6. Workowski KA, Berman SM. Centers for disease control and prevention sexually transmitted disease treatment guidelines. Clin Infect Dis. 2011;53(Suppl 3):S59-63. DOI: 10.1093/ cid/cir694

7. Shafir SC, Sorvillo FJ, Smith L. Current issues and considerations regarding trichomoniasis and human immunodeficiency virus in African-Americans. Clin Microbiol Rev. 2009;22(1):37-45. PMID: 19136432 DOI: 10.1128/CMR.00002-08

8. Wang CC, McClelland RS, Reilly M, Overbaugh J, Emery SR, Mandaliya $\mathrm{K}$, et al. The effect of treatment of vaginal infections on shedding of human immunodeficiency virus type 1. J Infect Dis. 2001;183(7):1017-22. PMID: 11237825 DOI: $10.1086 / 319287$

9. Matini M, Rezaie S, Mohebali M, Maghsood A, Rabiee S, Fallah M, et al. Prevalence of Trichomonas vaginalis infection in Hamadan City, Western Iran. Iran J Parasitol. 2012;7(2):67-72. PMID: 23109948

10. Clark CG, Diamond LS. Methods for cultivation of luminal

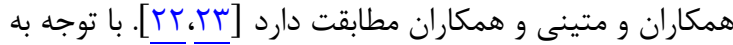
اينكه علائم انواع وازينيت، اغلب با يكديخر مشابه است و در آزمايشات بالينى، افتراق قطعى آن بهطور گسترده امكانيذير

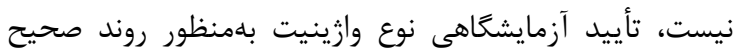
درمان بيماران ضرورى بهنظر مئرسد. علاوهبراين، نتايج آزمون حساسيت دارويى بر انگل نشان نهان داد كه تمامى ايزولههاى جداشده تريكوموناس وازيناليس از افراد آلوده، به داروى مترونيدازول حساس بوده و داراى ميانخين حداقل غلظت مهاركنندگى / | ا ميكروگرم در ميلىليتر هستند. در ارتباط با مطالعاتى كه براى تعيين حساسيت دارويى انجام شده است، مىتوان به مطالعه متينى و همكاران ( • وץ|) كه در مورد •ه ايزوله بلهستآمده از شهرهاى تهران، همدان و و تويسر كان صورت گرفت، اشاره نمود كه در آن ميانگين حداقل غلظت مهاركنندگى مترونيدازول برابر با ب/9 ميكروگرم در ميلى ليتر بهدست آمد [V]]. اين :زئوهشخران در مطالعه ديخرى كه در ارتباط با ايزوله جداشده در قروه كردستان انجام دادند، ميانگين حداقل غلظت مهاركنـدگى مترونيدازول را معادل ميكروگرم در ميلىليتر گزارش نمودند [Yr]]. در اين راستا، در

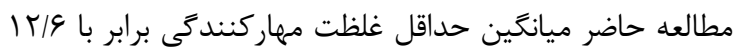
ميكروگرم ميلىليتر تعيين گَرديد كه اين تفاوت ميانگين در حساسيت دارويى مىتواند ناشى از وجود يكى ايزوله با تحمل بالاتر به دارو و همجنين تعداد كمتر ايزولههاى مورد بررسى در اين

parasitic protists of clinical importance. Clin Microbiol Rev. 2002;15(3):329-41. PMID: 12097242

11. Meingassner JG, Havelec L, Mieth H. Studies on strain sensitivity of Trichomonas vaginalis to metronidazole. $\mathrm{Br} \mathrm{J}$ Vener Dis. 1978;54(2):72-6. PMID: 305807

12. Schwebke JR, Barrientes FJ. Prevalence of Trichomonas vaginalis isolates with resistance to metronidazole and tinidazole. Antimicrob Agents Chemother. 2006;50(12):420910. PMID: 17000740 DOI: $10.1128 /$ AAC.00814-06

13. Matini M, Maghsood AH, Mohebali M, Rabiee S, Fallah M, Rezaie $\mathrm{S}$, et al. In vitro susceptibility of Iranian isolates of Trichomonas vaginalis to Metronidazole. Iran J Parasitol. 2016;11(1):46-51. PMID: 27095968

14. Esmaeili M, Haji AM, Asgardoon G, Ghadimi R, Baleghi M, Zahed PY. Effect of risk factors on low birth weight neonates. J Babol Univ Med Sci. 2004;6(2):18-24. [Persian]

15. Schwebke JR, Burgess D. Trichomoniasis. Clin Microbiol Rev. 2004;17(4):794-803. PMID: 15489349 DOI: 10.1128/ CMR.17.4.794-803.2004

16. Paulish-Miller TE, Augostini P, Schuyler JA, Smith WL, Mordechai E, Adelson ME, Gygax SE, Secor WE, Hilbert DW. Trichomonas vaginalis metronidazole resistance is associated with single nucleotide polymorphisms in the nitroreductase genes ntr4Tv and ntr6Tv. Antimicrob Agents Chemother. 2014;58(5):2938-43. PMID: 24550324 DOI: 10.1128/AAC.02370-13

17. Kassem HH, Majoud OA. Trichomoniasis among women with vaginal discharge in Benghazi city, Libya. J Egypt Soc Parasitol. 2006;36(3):1007-16. PMID: 17153709

18. Selvitopu A, Ozçelik S, Değerli S. The incidence of Trichomonas vaginalis in vaginal specimens from gynecologic patients. Turkiye Parazitol Derg. 2006;30(3): 175-7. PMID: 17160845

19. Garland SM, Tabrizi SN, Chen S, Byambaa C, Davaajav K. Prevalence of sexually transmitted infections (Neisseria gonorrhoeae, Chlamydia trachomatis, Trichomonas vaginalis 
and human papillomavirus) in female attendees of a sexually transmitted diseases clinic in Ulaanbaatar, Mongolia. Infect Dis Obstet Gynecol. 2001;9(3):143-6. PMID: 11516062 DOI: $\underline{10.1155 / \mathrm{S} 1064744901000254}$

20. Menéndez C, Castellsagué X, Renom M, Sacarlal J, Quintó L, Lloveras B, et al. Prevalence and risk factors of sexually transmitted infections and cervical neoplasia in women from a rural area of southern Mozambique. Infect Dis Obstet Gynecol. 2010;2010:609315. PMID: 20706691 DOI: 10.1155/2010/609315

21. Piperaki ET, Theodora M, Mendris M, Barbitsa L, Pitiriga V, Antsaklis A, et al. Prevalence of Trichomonas vaginalis infection in women attending a major gynaecological hospital in Greece: a cross-sectional study. $J$ Clin Pathol. 2010;63(3):249-53. PMID: 20203225 DOI: 10.1136/jcp. 2009.070920

22. Matini M, Golmoradi K, Maghsood AH, Fallah M. The prevalence of Trichomoniasis and metronidazole susceptibility of the isolates in Ghorveh city, year 2015. Sci J Hamadan Univ Med Sci. 2017;23(3):185-92. [Persian]

23. Akbari Z, Matini M. The study of Trichomoniasis in pregnant women attending Hamadan city health centers in 2015. Avicenna J Clin Microb Infect. 2017;4(2):e41533. DOI: 10.5812/ajcmi.41533

24. Nezhad MM, Jouybari LM, Sanagoo A, Haghdust Z, Mabasheri E. Prevalence of cervicovaginal infections in relationship with some factors in pap smear sampling. Iran J Obstet Gynecol Infertil. 2014;17(110):16-21. [Persian]
25. Ziaei Hezarjaribi H, Dalimi A, Ghasemi M, Ghafari R, Esmaeili S, Armat S, et al. Prevalence of common sexually transmitted diseases among women referring for pap smear in Sari, Iran. . J Mazandaran Univ Med Sci2013;22(11):19-24. [Persian]

26. Salmani R, Baghchesaraie H, Amini B. Prevalence of Trichomonas vaginalis infection among women referred to laboratories in Zanjan, 2010. J Res Dev Nurs Midwifery. 2012;9(1):69-75

27. Matini M, Rezaei H, Fallah M, Maghsood AH, Saidijam M, Shamsi-Ehsan T. Genotyping, drug susceptibility and prevalence survey of Trichomonas vaginalis among women attending gynecology clinics in Hamadan, Western Iran, in 2014-2015. Iran J Parasitol. 2017;12(1):29-37. PMID: 28761458

28. Nazari N, Zangeneh M. Moradi F, Bozorgomid A. Prevalence of Trichomoniasis among women in Kermanshah, Iran. Iran Red Crescent Med J. 2015;17(3):e23617. PMID: 26019904 DOI: $10.5812 /$ ircmj. 23617

29. Arbabi M, Fakhrieh Z, Delavari M, Abdoli A. Prevalence of Trichomonas vaginalis infection in Kashan city, Iran (20122013). Iran J Reprod Med. 2014;12(7):507-12. PMID: 25114674

30. Nourian A, Shabani N, Fazaeli A, Mousavinasab SN. Prevalence of Trichomonas vaginalis in pregnant women in Zanjan, northwest of Iran. Jundishapur $J$ Microbiol. 2013;6(8):e7258. 